\title{
Caracterización clínica y demográfica de la hospitalización psiquiátrica infantojuvenil en un hospital general
}

\author{
Clinical and demographic characteristics of children and \\ adolescents who were hospitalized for psychiatric reasons in a \\ general hospital
}

Viviana Duarte ${ }^{1}$, Lourdes Zelaya ${ }^{1}$

\section{RESUMEN}

Introducción: Se implementan actualmente nuevos modelos de hospitalización psiquiátrica infantojuvenil, para hacer frente a la creciente demanda de recursos de atención psiquiátrica intensiva requeridos por esta población. Objetivo: Identificar las características clínicas y demográficas de los niños y adolescentes con diagnóstico psiquiátrico hospitalizados en Salas de Pediatría de un Hospital General Pediátrico. Materiales y Métodos: Estudio descriptivo, retrospectivo de corte transversal, en base a historias clínicas de 180 casos hospitalizados en el periodo enero-agosto del año 2015.Variables: Edad, sexo, procedencia, motivo de ingreso, diagnóstico psiquiátrico, tipo de patología psiquiátrica, medicación, servicio desde el cual se indicó la evaluación psiquiátrica, días de hospitalización, seguimiento y derivación. Resultados: En el periodo de estudio se identificaron 180 pacientes hospitalizados con uno o más diagnósticos psiquiátricos, de los cuales $67.2 \%$ eran del sexo femenino. La mediana de la edad fue de 13 años. De un total de 387 diagnósticos constatados, los seis diagnósticos psiquiátricos más frecuentes fueron: los diferentes tipos de maltrato infantil $(42,89 \%)$, los trastornos depresivos $(21,71 \%)$, los intentos suicidas $(8,27 \%)$, el trastorno adaptativo $(4,39 \%)$ y el trastorno por estrés post traumático (4,13\%). El 56\% presentó comorbilidad de dos o más diagnósticos psiquiátricos. La internación fue indicada principalmente desde las Salas de Urgencias (67,3\%). El 54,4\% (98/180) estuvo hospitalizado de 1 a 3 días. En el 47,2\% de los casos se indicó psicofarmacoterapia. Conclusión: Se registró mayor frecuencia de diagnóstico psiquiátrico en adolescentes y en el sexo femenino. La sospecha de abuso sexual infantil representó un frecuente motivo de ingreso. Las distintas formas de maltrato Infantil, los trastornos depresivos y los intentos suicidas, al constituir los diagnósticos psiquiátricos

\section{ABSTRACT}

Introduction: Currently, new models of child and adolescent psychiatric hospitalizations are implemented to meet the increasing demands of resources for intensive psychiatric attention, due to the changes of mental health assistance necessities in this population. Objective: To identify the clinical and demographical characteristics of children and adolescents with psychiatric diagnosis hospitalized in Pediatric Wards of a General Pediatric Hospital. Materials and Methods: Descriptive, retrospective, cross-sectional study, on the basis of clinical histories of 180 hospitalized cases during January-August 2015. Variables: age, sex, origin, cause of admission, psychiatric diagnosis, type of psychiatric disorder, medication, hospital department from which psychiatric assessment was required, period of hospitalization, followup and referral. Results: During the study period 180 hospitalized patients with one or more psychiatric diagnosis were identified, from which $67,2 \%$ were female. The median age was 13 years. A total of 387 psychiatric diagnoses were documented, the six most frequently reported among them were the different types of child mistreatment $(42,89 \%)$, depressive mood disorders $(21,71 \%)$, suicide attempts $(8,27 \%)$, adjustment disorder $(4,39 \%)$ and post-traumatic stress disorder $(4,13 \%)$. Comorbidity of two or more psychiatric diagnoses was reported in $56 \%$ of patients. The hospitalization was indicated mainly from the Emergency Room (67,3\%), 54,4\% $(98 / 180)$ were hospitalized from 1 to 3 days. Pharmacotherapy was required in $47,2 \%$ of the cases. Conclusion: Psychiatric diagnoses were more frequently found in adolescents and in females. Sexual abuse suspicion represents a frequent reason of admission. The different types of child mistreatment, depressive mood disorders and suicide attempts are the most common

\footnotetext{
${ }^{1}$ Hospital General Pediátrico Niños de Acosta Ñu. Departamento de Salud Mental. San Lorenzo, Paraguay.

Correspondencia: Viviana Duarte Correo: viv6du@hotmail.com

Conflicto de interés: Los autores declaran no poseer conflicto de interés

Recibido: 13/05/2019 Aceptado: 18/07/2019

DOI: https://doi.org/10.31698/ped.46022019004

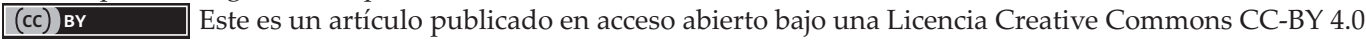

90 Pediatr. (Asunción). 2019; 46(2):90-96 (mayo - agosto) 
más frecuentes, requieren parámetros de evaluación y tratamiento específicos y efectivos para su adecuado manejo multidisciplinario. Las Salas de Urgencias Pediátricas cumplen un importante rol como puerta de entrada de estos pacientes al sistema de salud y al continuum de servicios de atención a la salud mental.

Palabras claves: Hospitalización psiquiátrica, psiquiatría de niños y adolescentes, psiquiatría de enlace pediátrico.

\section{INTRODUCCIÓN}

A nivel mundial 10 al 20\% de los niños y adolescentes presentan trastornos mentales. La mitad de las patologías mentales comienzan antes de los 14 años y tres cuartos de las mismas antes de los 24 años $^{(1)}$. De acuerdo a un informe de la OMS del 2018, en las Américas la brecha del tratamiento de los trastornos mentales infantojuveniles es alarmante y oscila del 64\% en Puerto Rico y EEUU al 86\% en México. En lo que respecta a los trastornos mentales severos se registró una brecha del tratamiento superior al 50\% (Chile 59,4\%-52,6\% EEUU). En Sudamérica 4,8\% de los Años de Vida Ajustados por Discapacidad (AVAD) se deben a trastornos mentales y por abuso de sustancias $^{(2)}$. Según lo señalado por un estudio reciente los niños con historia de eventos familiares adversos (Maltrato físico, abuso sexual, violencia intrafamiliar) tienen una probabilidad incrementada de utilización de servicios de salud mental ${ }^{(3)}$. En cuanto a la demanda de hospitalización se estima que en EEUU $10 \%$ de las internaciones pediátricas corresponden a pacientes que ingresan debido a un trastorno mental primario ${ }^{(4)}$. Las hospitalizaciones psiquiátricas, dirigidas a esta proporción de la población infantojuvenil con trastornos mentales, deben tener como propósito la estabilización del paciente y evitar el daño a otros o a el mismo. Se implementan actualmente nuevos modelos de hospitalización psiquiátrica infantojuvenil, para hacer frente a la creciente demanda de recursos de atención psiquiátrica intensiva, estos modelos tienen como premisa principal la hospitalización breve con énfasis en la estabilización aguda de los síntomas y el cambio necesario para continuar tratamiento ambulatorio ${ }^{(5)}$.

Los datos epidemiológicos citados aportan al desarrollo de programas y enfoques efectivos de psychiatric diagnoses, therefore, requiring specific and effective evaluation and treatment parameters for an adequate multidisciplinary approach. The Pediatric Emergency Rooms represent the entrance door of these patients to the health system and to the continuum of mental health care services.

Keywords: Psychiatric Hospitalization, child and adolescent psychiatry, pediatric consultation-liaison psychiatry.

tratamiento de niños y adolescentes hospitalizados por causa psiquiátrica, los cuales deberían considerar las recomendaciones emanadas de la Organización Mundial de la Salud con respecto a la incorporación de la atención a la salud mental en los servicios generales de salud, dentro de los cuales los hospitales generales representan un componente fundamental ${ }^{(6)}$.

El objetivo del presente estudio fue determinar las características clínicas de los niños y adolescentes que, durante su ingreso en un Hospital Pediátrico de la ciudad de San Lorenzo, Paraguay, tuvieron diagnostico psiquiátrico.

\section{MATERIALES Y MÉTODOS}

Estudio observacional, descriptivo de corte trasversal, retrospectivo. Se revisaron historias clínicas de pacientes de 0 a 18 años, con diagnóstico de patología psiquiátrica, ingresados en Salas de Pediatría del Hospital General Pediátrico “Niños de Acosta $\tilde{\mathrm{Nu}}$ ", en el periodo de enero a agosto del 2015. Las variables estudiadas fueron: Características demográficas: edad, sexo y procedencia. Características clínicas: Motivo de ingreso, diagnóstico psiquiátrico, patología psiquiátrica o secundaria, medicación psicofarmacológica, servicio desde el cual se indicó el ingreso o interconsulta, días de hospitalización, tipo de seguimiento, derivación externa.

El cálculo del tamaño muestral estableció que para conseguir una precisión del 3\% en la estimación de una proporción con un intervalo de confianza del $95 \%$ para poblaciones finitas de 260 , fue necesario incluir 173 pacientes. Los datos fueron analizados en el sistema SPSSv20. 
Los diagnósticos se realizaron en base a evaluaciones clínicas teniendo en cuenta los criterios diagnósticos del Manual Diagnóstico y Estadístico de los Trastornos Mentales Cuarta Edición Revisada (DSM -IV-TR) y la codificación de acuerdo a la Décima Edición de la Clasificación Internacional de Enfermedades (CIE 10). Todos los diagnósticos e intervenciones clínicas fueron realizadas por psiquiatras y psicólogos del Dpto. de Salud Mental entrenados en el manejo de trastornos mentales en la infancia y la adolescencia. Las indicaciones de internación y permanencia en Sala en pacientes hospitalizados por Trastornos psiquiátricos primarios fueron realizadas por Psiquiatras.

Se utilizó la estadística descriptiva para el análisis de las variables. Para variables cuantitativas de acuerdo a su distribución se utilizaron medias con desvíos standard o medianas con rangos. Las variables cualitativas se expresaron en porcentajes.

El protocolo fue aprobado por el Comité institucional de Investigación y Ética de la Investigación, que liberó el consentimiento informado.

\section{RESULTADOS}

En el periodo de estudio fueron hospitalizados 7042 pacientes, de los cuales el 2,5\% (180/7042) tuvieron diagnóstico psiquiátrico, y fue la población analizada.

Los datos demográficos se detallan en la Tabla 1. La mediana de la edad fue de 13 años.

Tabla 1. Datos demográficos de la población $(\mathrm{n}=180)$.

\begin{tabular}{|lcc|}
\hline Característica & $\mathbf{N}^{\circ}$ & $\%$ \\
\hline Grupo etario & & 1,1 \\
\hline Recién nacido & 2 & 7,8 \\
\hline Lactante & 14 & 10,0 \\
\hline Pre-Escolar & 17 & 22,8 \\
\hline Escolar & 38 & 58,3 \\
\hline Adolescente & 109 & \\
\hline Sexo & & 32,8 \\
\hline Masculino & 59 & 67,2 \\
\hline Femenino & 121 & \\
\hline Procedencia & & 69,4 \\
\hline Departamento Central & 125 & 7,8 \\
\hline San Pedro & 14 & 4,4 \\
\hline Caaguazú & 8 & 18,4 \\
\hline Otros departamentos & 33 & \\
\hline
\end{tabular}

Los servicios donde se identificaron los casos que requirieron ingreso psiquiátrico o evaluación psiquiátrica en paciente ya hospitalizados por otras causas se detallan en la Tabla 2.

Tabla 2. Servicio donde se indicó el ingreso psiquiátrico o la evaluación psiquiátrica $(\mathrm{n}=180)$.

\begin{tabular}{lcc}
\hline Salas & $\mathbf{N}^{\circ}$ & $\mathbf{\%}$ \\
\hline Urgencias & 121 & $67,3 \%$ \\
Salud Mental & 2 & $1,1 \%$ \\
Medicina Interna & 39 & $21,7 \%$ \\
Terapia Intensiva Pediátrica & 8 & $4,4 \%$ \\
Cuidados Intensivos Cardiológicos & 4 & $2,2 \%$ \\
Oncología & 4 & $2,2 \%$ \\
Terapia Intermedia & 2 & $1,1 \%$ \\
Totales & $\mathbf{1 8 0}$ & $\mathbf{1 0 0} \%$ \\
\hline
\end{tabular}

Los servicios donde se identificaron los casos que requirieron ingreso psiquiátrico o evaluación psiquiátrica en paciente ya hospitalizados por otras causas se detallan en la Tabla 2.

Del total de pacientes con diagnóstico psiquiátrico, $74,4 \%$ (134/180) fueron ingresados o requirieron de interconsulta hospitalaria debido a patología psiquiátrica o trastornos psiquiátricos primarios, los restantes (46/180), fueron ingresados debido a patología médica y durante la hospitalización desarrollaron trastornos psiquiátricos secundarios.

En la Tabla 3 se observan los Motivos de Ingreso de los 134 pacientes con Trastorno Psiquiátrico Primario.

Tabla 3. Motivos de Ingreso en pacientes con Trastornos Psiquiátricos Primarios. $(\mathrm{n}=134)$.

\begin{tabular}{lcc}
\hline Motivo de ingreso o interconsulta & N $^{\circ}$ & \% \\
\hline Sospecha o testimonio de abuso sexual & 28 & 20,9 \\
Queja somática sin causa orgánica & 17 & 12,7 \\
\hline Intento suicida & 17 & 12,7 \\
Síntomas depresivos & 12 & 9,0 \\
\hline Sospecha de maltrato infantil & 12 & 9,0 \\
Negligencia en los cuidados & 11 & 8,2 \\
Síntomas conversivos & 9 & 6,7 \\
Síntomas psicóticos & 6 & 4,5 \\
Síntomas de ansiedad & 6 & 4,5 \\
Consumo de sustancias & 5 & 3,7 \\
Agresividad & 4 & 3,0 \\
Maltrato físico & 3 & 2,2 \\
Problema del vínculo & 2 & 1,5 \\
Enfermedad Orgánica crónica & 1 & 0,7 \\
\hline Trastorno de conducta & 1 & 0,7 \\
\hline Total & $\mathbf{1 3 4}$ & $\mathbf{1 0 0}$ \\
\hline
\end{tabular}


Las patologías médicas que motivaron la internación de los 46 pacientes con Trastornos Psiquiátricos Secundarios se detallan en la Tabla 4.

Tabla 4. Motivos de ingreso (enfermedad medica) en pacientes con trastornos psiquiátricos secundarios.

\begin{tabular}{lcc}
\hline Enfermedad Médica & $\mathbf{N}^{\circ}$ & $\%$ \\
\hline Epilepsia & 12 & $26 \%$ \\
\hline Diabetes Mellitus & 8 & $17,40 \%$ \\
\hline Postoperatorio & 5 & $10,87 \%$ \\
\hline Patología Oncológica & 5 & $10,87 \%$ \\
\hline Infección & 4 & $8,70 \%$ \\
\hline Cardiopatía & 4 & $8,70 \%$ \\
\hline Lupus Eritematoso Sistémico & 2 & $4,35 \%$ \\
\hline Artritis Reumatoidea Juvenil & 2 & $4,35 \%$ \\
\hline Púrpura Trombocitopenia & 2 & $4,35 \%$ \\
\hline Otras & 3 & $4,41 \%$ \\
\hline Total & $\mathbf{4 6}$ & $\mathbf{1 0 0 , 0 0 \%}$ \\
\hline
\end{tabular}

El 56\% (121/180) presentó comorbilidad de dos o más diagnósticos psiquiátricos, por consiguiente, el total diagnósticos fue de 387. La frecuencia de los mismos se detalla en la Tabla 5.

Tabla 5. Diagnósticos psiquiátricos en pacientes hospitalizados. $(\mathrm{n}=387)$.

\begin{tabular}{|lcc|}
\hline Diagnóstico & $\mathbf{N}^{\circ}$ & $\mathbf{\%} \mathbf{( n = 3 8 7 )}$ \\
\hline Maltrato Infantil & 166 & $42,89 \% 2$ \\
\hline Trastorno Depresivo & 84 & $1,71 \%$ \\
\hline Intento suicida & 32 & $8,27 \%$ \\
\hline Trastorno adaptativo & 17 & $4,39 \%$ \\
\hline Trastorno por Estrés Post Traumático & 16 & $4,13 \%$ \\
\hline Trastorno de pánico & 12 & $3,10 \%$ \\
\hline Epilepsia & 12 & $3,10 \%$ \\
\hline Trastorno conversivo & 10 & $2,58 \%$ \\
\hline Delirium & 5 & $1,29 \%$ \\
\hline Abuso de sustancias & 5 & $1,29 \%$ \\
\hline Retraso mental & 5 & $1,29 \%$ \\
\hline Trastorno Bipolar & 3 & $0,78 \%$ \\
\hline Trastorno por Estrés Agudo & 3 & $0,78 \%$ \\
\hline Trastorno del aprendizaje & 3 & $0,78 \%$ \\
\hline Trastorno alimentario & 2 & $0,52 \%$ \\
\hline Trastorno Del Lenguaje & 2 & $0,52 \%$ \\
\hline Trastorno del Espectro Autista & 2 & $0,52 \%$ \\
\hline Trastorno Psicótico de etiología médica & 1 & $0,26 \%$ \\
\hline Trastorno afectivo orgánico & 1 & $0,26 \%$ \\
\hline Trastorno mental orgánico no especificado & 1 & $0,26 \%$ \\
\hline Abuso de alcohol & 1 & $0,26 \%$ \\
\hline Trastorno Psicótico Agudo & 1 & $0,26 \%$ \\
\hline Trastorno Obsesivo Compulsivo & 1 & $0,26 \%$ \\
\hline Trastorno de Personalidad & $0,26 \%$ \\
\hline Encopresis & 1 & $0,26 \%$ \\
\hline Total & $\mathbf{3 8 7}$ & $\mathbf{1 0 0} \%$ \\
\hline *Varios pacientes presentaron más de un diagnóstico psiquiátrico. & \\
\hline
\end{tabular}

El Maltrato Infantil englobó a cinco diferentes diagnósticos de acuerdo a los tipos de maltrato, los cuales frecuentemente se presentaron en comorbilidad, por lo tanto, los 110 pacientes con Maltrato Infantil presentaron dichos diagnósticos según las frecuencias detalladas a continuación: Abuso Sexual 25,9\% (43/166), Negligencia en los Cuidado 25,9\% (43/166), Maltrato Psicológico 19,3\% (32/166), Violencia Intrafamiliar 17,5\% (29/166), Maltrato Físico 11,4\% (19/166).

Los Trastornos Depresivos, el segundo diagnóstico más frecuente $(n=84)$ se dividieron de acuerdo al grado de severidad en cuatro subdiagnósticos según se detalla a continuación en orden de frecuencia: Trastorno Depresivo Moderado 39,3\%, Trastorno Depresivo Severo 27,4\%, Trastorno Depresivo Leve 20,2\% y Trastorno Depresivo con síntomas psicóticos 13,1\%.

Los intentos suicidas, tercer diagnóstico más frecuente fueron desglosados en cuatro subdiagnósticos según el método autolítico según se observa en la Tabla 6.

Tabla 6. Intentos suicidas según método de autoagresión ( $\mathrm{n}=32$ ).

\begin{tabular}{lcc}
\hline Método & $\mathbf{N}^{\circ}$ & $\mathbf{\%}$ \\
\hline Intento Suicida por Objeto Cortante & 15 & $46,9 \%$ \\
Intento Suicida por Ingesta de Medicación & 12 & $37,5 \%$ \\
\hline Intento Suicida por Ahorcamiento & 3 & $9,4 \%$ \\
Intento Suicida Por Electrocución & 2 & $6,3 \%$ \\
Total & $\mathbf{3 2}$ & $\mathbf{1 0 0 \%}$ \\
\hline
\end{tabular}

En el subgrupo compuesto por los 46 pacientes hospitalizados por patologías medicas se determinaron un total de 72 diagnósticos psiquiátricos (varios pacientes presentaron comorbilidad), de los cuales los más frecuentes fueron las distintas formas de maltrato infantil $26,3,7 \%$, Trastornos depresivos $23,6 \%$ y el trastorno adaptativo $19,4 \%$.

\section{E1 47,2\%(85/180) recibió tratamiento} psicofarmacológico.

Teniendo en cuenta el tipo de seguimiento realizado, los 177 pacientes que al alta estaban vivos (3 pacientes obitaron debido a patologías médicas) se dividieron en tres subgrupos: Ambulatorio (en el hospital donde se realizó el estudio) $88,7 \%$, Derivación externa 9,6\% y sin seguimiento (debido a alta sin consentimiento médico) $1,7 \%$. 
Las derivaciones externas $(\mathrm{n}=17)$ se realizaron a los siguientes dispositivos de tratamiento: Servicios de Salud Mental Comunitarios 75\%, Centro Nacional de Control de Adicciones 12,5\% y Hospital Psiquiátrico $12,5 \%$.

\section{DISCUSIÓN}

El porcentaje de diagnóstico psiquiátrico en la población hospitalizada analizada en el presente estudio fue ligeramente superior al obtenido por Mapelli y cols.quien, en el año 2015, reportó hospitalizaciones psiquiátricas en el 1,9\% de los pacientes de las salas de emergencias de un hospital pediátrico en Canadá ${ }^{(7)}$. En otro estudio realizado en un hospital pediátrico de Córdoba Argentina se encontró patologías psiquiátricas en el 1,26\% ${ }^{(8)}$.

En el presente estudio las hospitalizaciones por causa psiquiátrica fueron indicadas principalmente para el manejo de pacientes con trastornos graves recepcionados en el servicio de Urgencias. Estos resultados coinciden con lo referido en la bibliografía, de que, los departamentos de emergencias son la primera línea de provisión de servicios de atención a problemas de salud mental de la infancia y constituyen la principal puerta de entrada de estos pacientes al sistema de salud ${ }^{(9)}$. La escasez de recursos de salud mental comunitarios o la dificultad para el acceso a los mismos podría explicar estos hallazgos ${ }^{(7)}$.

La mayor frecuencia de participantes del sexo femenino, encontrada en este trabajo cual concuerda con lo reportado por Pupo y cols. en un Hospital Pediátrico de Cuba quienes en el 2018 encontraron cifras del $63 \%{ }^{(10)}$. Por otro lado, Bella y cols. en el Hospital de Niños de de la Santísima Trinidad de Córdoba (Argentina) en el 2016 no encontró diferencias en el sexo en una población de 0 a 15 años $^{(8)}$.

Los 4 motivos de ingreso más frecuentemente encontrados en esta investigación coinciden con los obtenidos por Bella y cols. ${ }^{(8)}$ en el 2016, quienes encontraron que las tres causas más frecuentes de hospitalización fueron los problemas de salud relacionados con la violencia: la sospecha de maltrato Infantil 44,2\%, comportamientos suicidas $18,7 \%$, la sospecha de abuso sexual $10,5 \%$ y el abuso de sustancias $6,8 \%$. Otro estudio realizado en un hospital terciario de Canadá, en una muestra de pacientes de 0 a 17 años ingresados a sala de urgencias, reportó que los principales motivos de ingreso fueron los síntomas depresivo- ansiosos y la ideación suicida representando el 50\% de los casos. Sin embargo, en el mismo no fueron incluidas causas psicosociales de ingreso como las relacionadas al maltrato infantil o abuso sexual, lo cual podría explicar las diferencias en relación a los resultados obtenidos en el presente estudio ${ }^{(7)}$.

La presencia de trastornos psiquiátricos secundarios, relacionados con enfermedades médicas crónicas tal como se reporta, ha sido corroborada por otros autores $^{(11)}$. En lo que respecta particularmente a la epilepsia como factor de riesgo para el desarrollo de síntomas psiquiátricos Bilgiç y cols. ${ }^{(12)}$ postularon en el 2018 que la epilepsia está asociada a un estado psiquiátrico deficiente en la infancia. En relación a la Diabetes Mellitus y su comorbilidad con trastornos psiquiátricos, Sztein y Lane en el 2009 analizaron una población de niños hospitalizados por enfermedades crónicas y concluyeron que los niños hospitalizados por asma, diabetes mellitus tipo I y diabetes mellitus tipo II tenían mayores probabilidades de ser dados de alta hospitalaria con un trastorno del humor o de ansiedad, con respecto a los otros niños dados de alta $^{(13)}$. Estos datos sugerirían que niños y adolescentes con estas enfermedades médicas deberían ser considerados una población de alto riesgo para el desarrollo de trastornos mentales secundarios y se beneficiarían con la aplicación de estrategias de screening para el diagnóstico precoz de los mismos.

Analizando los diferentes diagnósticos psiquiátricos encontrados predominaron los diferentes tipos de maltrato infantil, los trastornos depresivos y los intentos suicidas, los cuales, por su elevada frecuencia, requerirían parámetros de evaluación y tratamiento específicos y efectivos para su adecuado manejo multidisciplinario. De la Barra y García ${ }^{(5)}$ en el 2009, en un hospital general privado de Chile encontraron que los diagnósticos más frecuentes fueron los intentos suicidas (25\%), los trastornos afectivos $(21,2 \%)$ y las psicosis $13 \%$. Estas diferencias podrían atribuirse a diferencias en las características de ambos servicios (hospital general pediátrico 
público/hospital general privado) y a razones metodológicas como la no inclusión de diagnósticos psicosociales como el abuso sexual, el maltrato psicológico $u$ otros tipos de maltrato infantil en estudio arriba mencionado. Estos resultados, no obstante, son congruentes con la literatura en lo referente a que el maltrato, físico, sexual, emocional, así como las distintas formas de negligencia están asociados un riesgo incrementado de psicopatología en todas las etapas del desarrollo ${ }^{(14)}$. En el 2014 Keeshin y cols., quienes realizaron una revisión sistemática de 1433 fichas médicas correspondientes a hospitalizaciones psiquiátricas de niños y adolescentes que tuvieron lugar en un periodo de 10 meses. Los resultados mostraron que los pacientes con antecedentes de maltrato físico y sexual tenían mayor probabilidad de ser diagnosticados de múltiples trastornos de acuerdo al manual al DSM IV-TR comparados con los niños y adolescentes hospitalizados que no habían sufrido maltrato ${ }^{(15)}$.

Por otro lado Esmaeeli y Sayar, en el 2014 detectaron Trastornos depresivos en diferentes grados de severidad en el $63 \%$ de una muestra de pacientes hospitalizados de 8 a 16 años, con enfermedades médicas agudas, enfermedades médicas crónicas y cáncer, sin embargo, este estudio no incluyó la pesquisa de diagnósticos psicosociales como el maltrato infantil, tal como se realizó en este estudio $^{(16)}$.

Las frecuencias de los diferentes tipos de maltrato Infantil encontradas en este estudio difieren de las descriptas por Ulloa y Navarro ${ }^{(17)}$ en su estudio del 2011, en el cual reportaron las siguientes las frecuencias: maltrato emocional $77 \%$, maltrato físico $41 \%$, negligencia $8 \%$ y abuso sexual $24 \%$, estas discrepancias podrían atribuirse a que dicho estudio fue realizado en una muestra de adolescentes de 13 a 17 años de ambos sexos, usuarios de los servicios de consulta externa, urgencias y hospitalización de un Hospital Psiquiátrico Infantil, mientras que el presente trabajo incluyó pacientes de menor edad además de los adolescentes.

En relación al intento de suicidio, fue el tercer diagnostico en orden de frecuencia. El daño autoinfligido por objeto cortante fue el método más frecuente, seguido del intento suicida por ingesta de medicación, el intento suicida por ahorcamiento y el intento suicida por electrocución. Estas frecuencias son diferentes de las halladas en Hospitales Pediátricos de E.E.U.U en el estudio de Sulyman y cols. ${ }^{(18)}$ en el que la ingesta de medicación fue el método más frecuente $(70 \%)$, así mismo difieren de las observadas por Pupo Cols. ${ }^{(10)}$ en una muestra de pacientes de 0 a 18 años hospitalizados en el servicio de psiquiatría infantil de un Hospital General Pediátrico cubano en quienes también reportaron como método más frecuente a la ingestión de sustancias toxicas con fines suicidas con cifras del $70,3 \%(365 / 519)^{(16)}$. Esta diferencia se atribuye a la denominación como intento suicida, utilizada en el hospital donde se realizó el estudio, para las lesiones superficiales autoinfligidas por objetos cortantes o "cutting" en concordancia con la definición de la Organización Mundial de la Salud ${ }^{(19)}$. Otros autores como Grandclerc y cols. ${ }^{(20)}$ en su estudio del 2016, las consideran como autolesiones no suicidas.

La frecuencia de indicación de tratamiento farmacológico y la duración de las hospitalizaciones fueron similares a las reportadas por otros autores ${ }^{(5)}$. Este estudio presenta como limitación el hecho de que, al describir el mismo los ingresos o las interconsultas psiquiátricas en el contexto de un hospital general, con criterios de admisión adaptados para ese fin, los resultados no se podrían generalizar para describir frecuencias de variables clínicas en centros monovalentes (ingresos psiquiátricos en dispositivos especializados), los cuales son requeridos para el tratamiento de una proporción de pacientes con psicopatología severa cuyas descompensaciones agudas requieren de manejo en dispositivos de mayor complejidad.

\section{CONCLUSIONES}

La frecuencia de presentación de diagnósticos psiquiátricos en pacientes hospitalizados en un hospital general pediátrico fue del 2,5\%. Los motivos de ingreso más frecuentes fueron el abuso sexual infantil, los síntomas psicosomáticos, la conducta suicida, y los síntomas depresivos. Los diagnósticos más frecuentes fueron las distintas formas de maltrato infantil, los trastornos depresivos en diferentes grados de severidad y los intentos suicidas. Los diagnósticos correspondieron a 
patología psiquiátrica primaria en el $74,4 \%$ de los casos. La patología psiquiátrica secundaria estuvo frecuentemente asociada a enfermedades medicas

\section{REFERENCIAS BIBLIOGRÁFICAS}

1. Shatkin JP, Balloge N, Belfer ML. Child and adolescent mental health policy worldwide: an update. International Psychiatry. Cambridge University Press; 2008; 5(4):81-4.

2. Organización Panamericana de la Salud. La carga de los trastornos mentales en la Región de las Américas. Washington: OPS; 2018.

3. Alcalá HE, Balkrishnan R. Mental Health Services in Childhood: The Role of Family Adversity. Public Health Reports. 2019; 134(2):180-88.

4. Bardach NS, Burkhart Q, Richardson LP. HospitalBased Quality Measures for Pediatric Mental Health Care. Pediatrics. 2018; 141(6):35-54.

5. De la Barra F, García R. Hospitalización psiquiátrica de niños y adolescentes II: Experiencia clínica en un hospital general privado. Rev. chil. neuro-psiquiatr. 2009; 47(3):238-243.

6. Organización Mundial de la Salud. Plan de acción sobre Salud Mental 2015-2020. [Internet] Washington: OMS; 2014. [Citado 3 de mayo de 2019]. Disponible en: https://www.paho.org/hq/dmdocuments/2015/plan-deaccion-SM-2014.pdf

7. Mapelli E, Black T, Doan Q. Trends in Pediatric Emergency Department Utilization for Mental HealthRelated Visits. J Pediatr. 2015; 167(4):905-10.

8. Bella M, Borgiattino V. Demand for hospitalization due to psychosocial causes in a pediatric hospital. Arch Argent Pediatr. 2016; 114(3):252-57.

9. Kalb L, Stapp E, Ballard E, Holingue C, Keefer A, Riley A. Trends in Psychiatric Emergency Department Visits Among Youth and Young Adults in the US. Pediatrics. 2019; 143(4):182-92.

10. Pupo L, Nogueras Y, Prada ME, Labrada D. Salud mental infanto juvenil, características de una problemática actual. Rev. electrón. Dr. Zoilo E. Marinello [Internet]. 2018[Citado 2 de mayo de 2019]; 43(6). Disponible en: http://www. revzoilomarinello.sld.cu/index.php/zmv/article/view/1687

11. Butler A, Van Lieshout RJ, Lipman EL. Mental disorder crónicas. La estadía hospitalaria fue breve y se requirió tratamiento psicofarmacológico en casi la mitad de la población analizada.

in children with physical conditions: a pilot study.BM] Open. 2018; 8(1)101-10.

12. Bilgiç A, Işık Ü, Çolak RS, Derin $H$, Çaksen $H$. Psychiatric symptoms and health-related quality of life in children with epilepsy and their mothers. Epilepsy \& Behavior. 2018; 80:114-121.

13. Sztein D, Lane W. Examination of the Comorbidity of Mental Illness and Somatic Conditions in Hospitalized Children in the United States Using the Kids' Inpatient Database 2009. Hospital Pediatrics. 2016; 6(3):126-134.

14. Zeanah CH, Humphreys KL. Child Abuse and Neglect. J Am Acad Child Adolesc Psychiatry. 2018; 57(9):637-644.

15. Keeshin B, Strawn J, Luebbe A, Saldaña S, Wehry A, DelBello $\mathrm{M}$, et al. Hospitalized youth and child abuse: a systematic examination of psychiatric morbidity and clinical severity. Child Abuse Negl. 2014;38(1):76-83.

16. Esmaeeli M, Erfani Sayar R, Saghebi A, Saghi E, Rahmani SH, Elmi S, et al. Screening for Depression in Hospitalized Pediatric Patients. Iran J Child Neurol. 2014; $8(1): 47-51$.

17. Ulloa R, Navarro G. Estudio descriptivo de la prevalencia y tipos de maltrato en adolescentes con psicopatología. Salud Mental. 2011;34(3):219-225.

18. Sulyman N, Kim MK, Rampa S, Allareddy V, Nalliah RP. Self Inflicted Injuries among Children in United States - estimates from a nationwide emergency department sample. PLoS ONE [Internet]. 2013[Citado 1 de mayo de 2019]; 8(7):e69874. Disponible en: https://www.ncbi.nlm. nih.gov/pmc/articles/PMC3715517/

19. World Health Organization. Preventing suicide: a global imperative [Internet]. Ginebra: WHO; 2014. [Citado 2 de mayo de 2019]. Disponible en: http://apps. who.int/ iris/bitstream/10665/131056/1/9789241564779_eng.pdf

20. Grandclerc S, De Labrouhe D, Spodenkiewicz M, Lachal J, Moro M. Relations between Nonsuicidal Self-Injury and Suicidal Behavior in Adolescence: A Systematic Review. PLoS ONE [Internet]. 2016 [Citado 3 de setiembre de 2018]; 11(4): e0153760. https://www.ncbi.nlm.nih.gov/pubmed/27089157 